\title{
Avaliação dos níveis álgicos de pacientes críticos em terapia intensiva, antes, durante e após sessão de fisioterapia respiratória: um estudo piloto
}

\author{
Evaluation of pain levels of critically ill intensive care unit patients, before, \\ during and after a session of chest physical therapy: a pilot study
}

Nathalya Tamara Costa Fermiano ${ }^{1}$, Odete Mauad Cavenaghi ${ }^{2}$, Juliana Rodrigues Correia ${ }^{2}$, Marcus Vinicius Camargo de Brito $^{2}$, Lucas Lima Ferreira ${ }^{2} \bowtie$

${ }^{1}$ Curso de Pós-graduação em Fisioterapia da Faculdade de Medicina de São José do Rio Preto (FAMERP). São José do Rio Preto, SP.

2 Unidade de Terapia Intensiva Geral do Hospital de Base da Fundação Faculdade Regional de Medicina (FUNFARME) / FAMERP de São José do Rio Preto.

São José do Rio Preto, SP.

\section{RESUMO}

OBJETIVOS: Avaliar os níveis álgicos de pacientes críticos, antes, durante e após uma intervenção de fisioterapia respiratória.

MÉTODOS: Estudo piloto realizado na Unidade de Terapia Intensiva de um hospital escola. Foram selecionados pacientes adultos entre 18 e 60 anos, sedados e em ventilação mecânica invasiva. Foram levantados dados sociodemográficos, clínicos e hemodinâmicos e aplicada a escala Behavioural Pain Scale para avaliar a dor em pacientes incapazes de serem avaliados pelo auto relato. O protocolo de fisioterapia consistiu em manobras de vibrocompressão manual em ambos os hemitórax e aspiração intratraqueal. As variáveis hemodinâmicas e a escala de dor foram analisadas em três momentos: imediatamente antes, no quinto minuto de intervenção e imediatamente após a sessão de fisioterapia. Foi aplicada estatística com teste Shapiro-Wilk e análise de variâncias (ANOVA). O nível de significância foi p $\leq 0,05$.

RESULTADOS: A amostra foi composta por 22 pacientes, a maioria do sexo masculino, com média de idade de $55 \pm 23,8$ anos. A doença mais frequente foi a pneumonia nosocomial. Não houve diferenças significativas nas variáveis hemodinâmicas e na avaliação da dor $(p=0,78)$ dos pacientes críticos em nenhum dos momentos avaliados.

CONCLUSÕES: Nesta amostra de pacientes críticos, internados em unidade de terapia intensiva e em ventilação mecânica invasiva, não foram detectadas alterações nos níveis de dor durante ou após uma intervenção de fisioterapia respiratória.

DESCRITORES: medição da dor; cuidados críticos; unidades de terapia intensiva; respiração artificial; especialidade fisioterapia; modalidades de fisioterapia.

\section{ABSTRACT}

AIMS: To evaluate pain levels of critically ill patients before, during, and after a chest physical therapy intervention.

METHODS: Pilot study conducted at the intensive care unit (ICU) of a teaching hospital. Sedated and mechanically ventilated adult patients aged 18 to 60 years were selected. Sociodemographic, clinical, and hemodynamic data were analyzed, and the Behavioral Pain Scale (BPS) scale was used to assess pain in patients unable to be evaluated by self-report questionnaires.

The physical therapy protocol consisted of manual vibrocompression maneuvers in both hemithoraces and of intratracheal aspiration. The hemodynamic variables and the BPS were analyzed in three periods: immediately before, in the fifth minute of intervention, and immediately after physical therapy. The Shapiro-Wilk test and ANOVA were used for the statistical analyses. The level of significance was $\mathrm{p} \leq 0.05$.

RESULTS: The sample consisted of 22 patients, most of them male, aged $55 \pm 23.8$ years. Nosocomial pneumonia the most prevalent pathology. There were no significant differences in hemodynamic variables and in pain assessment $(p=0.78)$ of the critically ill patients in any of the evaluated periods.

CONCLUSIONS: In this sample of critically ill and mechanically ventilated ICU patients, no alterations in pain levels were detected during or after chest physical therapy.

KEY WORDS: pain measurement; critical care; intensive care units; respiration, artificial; physical therapy specialty; physical therapy modalities. 
Abreviaturas: BPS, Behavioral Pain Scale; FC, frequência cardíaca; $\mathrm{FiO}_{2}$, fração inspirada de oxigênio; $\mathrm{FR}$, frequência respiratória; PA, pressão arterial; PAD, pressão arterial diastólica; PAM, pressão arterial média; PAS, pressão arterial sistólica; $\mathrm{SpO}_{2}$, saturação periférica de oxigênio; UTI, unidade de terapia intensiva.

\section{INTRODUÇÃO}

Os cuidados despendidos a pacientes críticos estão cada vez mais aprimorados e visam, não apenas, aumentar a sobrevida e permitir que os indivíduos possam ter uma reintegração social, como em alguns casos proporcionar o mínimo desconforto possível durante a permanência no ambiente hospitalar $[1,2]$. Um paciente é considerado crítico quando há risco iminente de vida por instabilidade hemodinâmica ou pelo déficit de função de um órgão ou sistema do organismo, necessitando de cuidado clínico imediato $[3,4]$.

A monitorização, durante 24 horas por dia, de parâmetros como hemodinâmica central, perfusão tecidual, temperatura, ventilação e estado nutricional é primordial nesses pacientes; sendo assim, a unidade de terapia intensiva (UTI) é um dos setores no ambiente hospitalar com maior complexidade, possuindo equipamentos de alta tecnologia e equipe multidisciplinar com conhecimentos e experiências para oferecer o suporte adequado $[5,6]$.

Durante a permanência na UTI, a dor se faz presente na maioria dos pacientes, ocorrendo tanto pela natureza da doença quanto pelos procedimentos que são realizados, muitas vezes de forma invasiva, provocando desconforto. Por isso, a compreensão sobre a experiência dolorosa e a identificação da dor pelos profissionais de saúde envolvidos com o paciente são importantes para que sejam tomadas medidas analgésicas [7]. Entretanto, evidências mostram que grande parte dos pacientes não recebe um adequado controle da dor, muitas vezes devido a métodos inadequados de avaliação e/ou intervenção insuficiente sobre as causas que a provocam [8].

A dor possui componentes psicológicos e subjetivos, sendo assim cada pessoa reage de forma particular. Um dos maiores desafios para os profissionais é a sua mensuração, por isso foram criadas diversas escalas com a finalidade de mensurar o quadro álgico e a partir daí promover a intervenção [9-11].

A avaliação da dor no ambiente de UTI é particularmente complexa, visto que grande parte dos instrumentos de avaliação da dor no adulto consistem no auto relato dos pacientes, que em situações críticas estão frequentemente incapazes ou impossibilitados de se comunicar verbalmente, principalmente por causa de intubação traqueal e alterações de consciência [12]. Com base nessa dificuldade, várias escalas estão sendo desenvolvidas para avaliar a dor em pacientes sob ventilação mecânica, como a Critical-Care Pain Observation Tool [13], a Pain Assessment and Intervention Notation Algoritm [14] e a Behavioral Pain Scale (BPS) [15].

Estudos recentes constataram que um dos importantes sinais vitais para avaliação da dor em pacientes críticos na UTI é o aumento da frequência cardíaca (FC), juntamente com aumento da pressão arterial (PA) devido à tensão que a dor provoca, estimulando o sistema nervoso autônomo, produzindo adrenalina, que por sua vez acarreta a constrição arteriolar [2].

A fisioterapia possui um aparato de técnicas e intervenções capazes de promover a preservação e a recuperação da funcionalidade do paciente, sendo uma peça primordial na equipe multidisciplinar da UTI. Entretanto, durante o atendimento, as próprias condutas fisioterapêuticas, como, por exemplo, aspiração traqueal e mudança de decúbito, podem provocar desconforto e dor [16, 17].

Estudos avaliando a dor em pacientes adultos na UTI são escassos. Foram encontradas evidências sobre mensuração da dor na pediatria e neonatologia com escalas específicas [18] e escalas validadas para avaliar o nível álgico de pacientes adultos [13-15], porém, não foram identificados estudos que avaliaram esta temática após intervenção de fisioterapia em terapia intensiva. Dessa forma, justifica-se a necessidade desta pesquisa pela relevância do assunto.

Sendo assim, o objetivo deste estudo foi avaliar os níveis de dor em pacientes adultos internados em UTI, sedados, sob ventilação mecânica invasiva, antes, durante e após uma intervenção de fisioterapia respiratória. Este estudo piloto foi desenvolvido para calcular o tamanho amostral necessário e avaliar a factibilidade dos procedimentos para realização de um ensaio clínico prospectivo a ser realizado pela mesma equipe de pesquisadores.

\section{MÉTODOS}

Foi realizado um estudo piloto de intervenção que incluiu pacientes internados pelo Sistema Único de Saúde na UTI Geral do Hospital de Base da Fundação Faculdade Regional de Medicina (FUNFARME)/ Faculdade de Medicina de São José do Rio Preto 
(FAMERP), em São José do Rio Preto, São Paulo. Foram definidos como participantes do estudo pacientes com idade entre 18 e 60 anos, de ambos os sexos, sedados, sob ventilação mecânica invasiva, com prescrição médica para atendimento fisioterapêutico e apresentando estabilidade hemodinâmica, definida como pressão arterial sistólica (PAS) $<200 \mathrm{mmHg}$ $>90 \mathrm{mmHg}$, saturação periférica de oxigênio $\left(\mathrm{SpO}_{2}\right)$ $>90 \%$, fração inspirada de oxigênio $\left(\mathrm{FiO}_{2}\right)<60 \%$ e frequência respiratória $(\mathrm{FR})<35$ movimentos por minuto. Foram excluídos pacientes que, durante o atendimento fisioterapêutico, começassem a apresentar instabilidade hemodinâmica, caracterizada por aumento ou diminuição em $20 \%$ dos parâmetros hemodinâmicos basais ( $\mathrm{FC}, \mathrm{PA}, \mathrm{FR}$ e $\mathrm{SpO}_{2}$ ). Além disso, foram excluídos pacientes que apresentassem indícios de morte encefálica, caracterizada por ausência de reflexos primitivos centrais.

Dados sociodemográficos e clínicos foram obtidos da ficha de admissão médica na UTI: nome, idade, sexo, peso e diagnóstico de admissão. Os dados hemodinâmicos, PAS, pressão arterial diastólica (PAD), pressão arterial média (PAM), FC, FR e $\mathrm{SpO}_{2}$, foram avaliados por meio do monitor multi paramétrico (IntelliVue MXZ700 ${ }^{\circledR}$ Philips, Amsterdã, Países Baixos).

Para avaliação da dor foi aplicada a escala BPS e foram avaliadas as variáveis hemodinâmicas de desfecho - PAS, PAD, PAM, FC e $\mathrm{SpO}_{2}$ - em três momentos: imediatamente antes, no quinto minuto de intervenção e imediatamente após a sessão de fisioterapia. As avaliações foram feitas pelo mesmo pesquisador que aplicou a intervenção fisioterapêutica.

A escala BPS, que é utilizada para avaliar pacientes sedados e inconscientes sob ventilação mecânica, ou seja, incapazes de ser avaliados pelo auto relato, consiste da avaliação de três aspectos: expressão facial, movimentos corporais e tolerância à ventilação mecânica. Cada indicador é classificado em quatro descrições do comportamento, indicando a ausência de dor (pontuação 1) ao máximo de dor (pontuação 4); dessa maneira, a pontuação final varia entre 3 (sem dor) e 12 pontos (dor máxima). O instrumento é de fácil reprodutividade, com tempo máximo de aplicação de dois a cinco minutos, sendo importante para nortear as condutas mais adequadas [12].

O protocolo de fisioterapia respiratória consistiu em manobras de vibrocompressão manual em ambos os hemitórax durante 10 minutos e aspiração intratraqueal com e sem instilação de soro fisiológico, com duração total de 20 minutos. Todas as intervenções foram realizadas pelo mesmo fisioterapeuta em todos os pacientes, os quais permaneceram em decúbito dorsal e com a cabeceira elevada a $30^{\circ}$ durante todo o procedimento [19].

O estudo foi aprovado pelo Comitê de Ética em Pesquisa da FAMERP sob parecer de número 1.148.529/2015. Foi obtido o termo de consentimento livre e esclarecido do parente mais próximo do paciente, de acordo com a Resolução de 466/12 do Conselho Nacional de Saúde e a Declaração de Helsinki de 1975, revisada em 1983.

Os resultados foram apresentados de forma descritiva em número absoluto, percentual, média e desvio padrão. Foi aplicada estatística inferencial composta por teste de Shapiro-Wilk para verificar a normalidade dos dados e análise de variâncias (ANOVA) para comparar as variáveis hemodinâmicas e o escore da escala BPS nos momentos analisados. O nível de significância adotado foi $p<0,05$. As análises estatísticas foram realizadas com o programa SPSS Statistics 17 para Windows.

\section{RESULTADOS}

Foram avaliados 22 pacientes, cujos dados estão descritos na Tabela 1. A doença mais frequente na amostra foi a pneumonia nosocomial.

Tabela 1. Características demográficas e clínicas dos 22 pacientes críticos internados na unidade de terapia intensiva e incluídos no estudo. São José do Rio Preto, SP, 2015.

\begin{tabular}{lcc}
\hline \multicolumn{1}{c}{ Variáveis } & Média (DP) & $\mathbf{n}(\%)$ \\
\hline Idade (anos) & $55,5 \pm 23,82$ & \\
Peso $(\mathrm{kg})$ & $63,75 \pm 18,53$ & \\
Sexo & & \\
$\quad$ Masculino & $15(65,21)$ \\
$\quad$ Feminino & $7(30,43)$ \\
Doença & \\
$\quad$ Pneumonia nosocomial & $6(26,0)$ \\
$\quad$ Choque Séptico & $4(17,3)$ \\
$\quad$ Poli trauma / TCE & $3(13,0)$ \\
$\quad$ DPOC & $3(13,0)$ \\
\hline
\end{tabular}

DP, desvio padrão; TCE, trauma crânio encefálico; DPOC, doença pulmonar obstrutiva crônica.

$\mathrm{Na}$ avaliação das variáveis FC, PAS, PAD, PAM e $\mathrm{SpO}_{2}$, não se observaram diferenças estatisticamente significativas entre os momentos imediatamente antes, no quinto minuto e imediatamente após a intervenção, em nenhum dos parâmetros (Tabela 2).

Não foram detectadas diferenças estatisticamente significativas nos níveis álgicos desse grupo de pacientes nos momentos analisados (Tabela 3 ). 
Tabela 2. Variáveis hemodinâmicas utilizadas para avaliação de dor, antes, durante e após uma única intervenção de fisioterapia respiratória em pacientes críticos internados na unidade de terapia intensiva, sob ventilação invasiva. São José do Rio Preto, SP, 2015.

\begin{tabular}{|c|c|c|c|c|}
\hline Variável & Momento 0 & Momento 1 & Momento 2 & $\mathbf{p}^{*}$ \\
\hline $\mathrm{FC}(\mathrm{bpm})$ & $101,86 \pm 12,43$ & $102,90 \pm 13,19$ & $102,68 \pm 12,46$ & 0,95 \\
\hline PAS (mmHg) & $126,22 \pm 25,31$ & $122 \pm 25,57$ & $124,95 \pm 27,17$ & 0,85 \\
\hline PAD (mmHg) & $67,27 \pm 12,94$ & $63,09 \pm 13,96$ & $65,77 \pm 14,73$ & 0,60 \\
\hline PAM (mmHg) & $83,71 \pm 16,16$ & $81 \pm 17,17$ & $84,33 \pm 18,51$ & 0,80 \\
\hline $\mathrm{SpO}_{2}(\%)$ & $95,61 \pm 3,51$ & $96,38 \pm 2,32$ & $96 \pm 3,47$ & 0,75 \\
\hline
\end{tabular}

Momento 0: imediatamente antes da intervenção; Momento 1: quinto minuto de intervenção; Momento 2: imediatamente após a intervenção. FC, frequência cardíaca; bpm, batimentos por minuto; PAS, pressão arterial sistólica; mmHg, milímetros de mercúrio; PAD, pressão arterial diastólica; PAM, pressão arterial média; $\mathrm{SpO}_{2}$, saturação periférica de oxigênio.

* ANOVA one way com pós-teste de Tukey.

Tabela 3. Escores da Behavioural Pain Scale antes, durante e após uma única intervenção de fisioterapia respiratória em pacientes críticos internados na unidade de terapia intensiva. São José do Rio Preto - SP, 2015.

\begin{tabular}{|ccc}
\hline Momento & Escala BPS & p* \\
\hline Momento 0 & $3,31 \pm 0,77$ & \\
\hline Momento 1 & $3,5 \pm 1,01$ & 0,78 \\
\hline Momento 2 & $3,40 \pm 0,79$ & \\
\hline
\end{tabular}

BPS, Behavioural Pain Scale.

Momento 0: imediatamente antes da intervenção; Momento 1: quinto minuto de intervenção; Momento 2: imediatamente após a intervenção.

* Teste de Dunn com pós-teste de Kruskal-Wallis.

\section{DISCUSSÃO}

Os resultados do presente estudo indicaram que não houve alteração significativa dos padrões hemodinâmicos utilizados para avaliação de dor ( $\mathrm{FC}, \mathrm{PA}$ e $\mathrm{SpO}_{2}$ ) com a intervenção da fisioterapia respiratória. O escore da escala BPS de dor, utilizada em três momentos específicos, avaliando expressão facial, movimentos de membros superiores e aceitação da ventilação mecânica, também não apresentou alterações significativas, mantendo o escore mínimo na maioria dos pacientes, o que indica que não ocorreu acentuação no nível da dor.

Mendes et al. [21] observaram, em uma UTI neonatal, que os principais sinais indicativos dos níveis álgicos foram face de dor, choro, movimentos excessivos de membros, taquicardia e queda da saturação, porém os autores salientam que a expressão facial permite a informação mais válida e sensível a respeito da natureza e da intensidade da dor. Já Freitas et al. [22], durante a avaliação comportamental de neonatos após procedimentos agressivos na UTI, verificaram que indicativos como expressão facial, choro excessivo, alteração no padrão respiratório e movimentos repetitivos de braços e pernas foram os mais observados. Isso sugere que essas variáveis presentes na escala BPS possam ser fidedignas para avaliar a dor também em pacientes adultos que não conseguem se comunicar.

Expressões comportamentais não foram observadas nos pacientes analisados no presente estudo. Cabe salientar que se tratou de uma população de adultos, diferente da maioria dos relatos apresentados na literatura, que se referem à população neonatal. Verificou-se escassez de dados na literatura acerca da investigação da dor no paciente crítico adulto durante intervenções de fisioterapia, o que justifica este estudo piloto.

A dor é um fenômeno multidimensional, envolvendo aspectos físico-sensoriais e emocionais. Não se trata apenas de um incômodo, mas consiste em complexas reações fisiológicas que acarretam imunossupressão, hipóxia tecidual, incremento no oxigênio consumido, maior esforço cardíaco, espasmos musculares e comprometimento na mecânica ventilatória [23].

A vias fisiológicas do estímulo doloroso incluem os nociceptores, que são terminações nervosas livres não especializadas responsáveis por captar os estímulos de dor por meio de vias aferentes e conduzir essas informações ao cérebro; isso acontece por meio da tradução dos estímulos sensoriais em impulsos nervosos, que por sua vez, o sistema nervoso interpreta como estímulo nocivo. A atividade desses receptores é modulada por várias substâncias químicas que são liberadas em virtude dos processos inflamatórios, traumáticos e isquêmicos, dentre as quais pode-se citar histamina, leucotrieno e substância P [24]. Compreender os mecanismos fisiológicos da dor é importante para entender o mecanismo de ação dos analgésicos: os opioides inibem os impulsos aferentes no sistema nervoso central; os anestésicos locais interrompem diretamente a condução do impulso doloroso; e os anti-inflamatórios não esteroides 
impedem a sensibilização dos nociceptores que ocorre nos processos inflamatórios [24].

Estudos demonstram que a fisioterapia respiratória provoca alterações hemodinâmicas momentâneas no paciente crítico $[25,26]$. No estudo de Moreira et al. [26] a intervenção fisioterapêutica promoveu alterações ventilatórias, como aumento da complacência pulmonar dinâmica e do volume corrente e redução da resistência do sistema respiratório; além dessas, aquele estudo também apontou para alterações hemodinâmicas como incremento da $\mathrm{SpO}_{2}$ e elevação imediata, porém não sustentada, da FC, em consequência da estimulação traqueal que provoca o aumento da atividade simpática, resultando em taquicardia. Contudo, tal alteração hemodinâmica não foi observada neste estudo, o que pode ser explicado pelo nível de sedação e analgesia em que se encontravam os pacientes e/ou pelo reduzido tamanho amostral.

O controle da dor é uma questão que abrange o interesse de diversos profissionais. A investigação minuciosa de suas características é fundamental na elaboração de estratégias, pois o paciente em ventilação mecânica deve ser avaliado constantemente quanto à dor e à sedação para otimizar a dose de medicamentos. Isso reduz a necessidade de suporte e de maior permanência em UTI [23]. Partindo desse pressuposto, o presente estudo aponta para algumas implicações clínicas, como a importância de que as medidas para alívio da dor sejam acompanhadas por uma equipe multiprofissional. É necessário que os profissionais que atuam em UTI, incluindo os fisioterapeutas, utilizem corretamente ferramentas para identificação da dor e, a partir disso, possam traçar ações efetivas com o objetivo de promover o conforto dos pacientes.

Este estudo apresenta algumas limitações, como o fato de que os medicamentos que estavam sendo administrados não foram utilizados como critério para inclusão ou exclusão do estudo, já que cada um possui uma via de ação diferente e promove um nível de sedação diferente; o reduzido tamanho da amostra; o mesmo avaliador realizar a intervenção e a avaliação, o que não permite uma avaliação mais criteriosa das reações do paciente durante a sessão. Além disso, por se tratar de uma avaliação única, as condições do paciente naquele momento poderiam não refletir o seu estado clínico mais constante. Uma avaliação a longo prazo poderia fornecer mais informações sobre os níveis álgicos nesses pacientes.

Mesmo considerando essas limitações, este estudo piloto foi útil por demonstrar que uma única intervenção de fisioterapia respiratória nas condições estudadas não causou alterações nos níveis álgicos desta amostra de pacientes adultos internados em UTI, sedados e sob ventilação mecânica invasiva.

\section{NOTAS}

\section{Apoio financeiro}

Este estudo não recebeu apoio financeiro de fontes externas.

Declaração de conflito de interesses

Os autores declaram não haver conflitos de interesses relevantes ao conteúdo deste estudo, informam ter tido acesso a todos os dados obtidos e assumem completa responsabilidade pela integridade dos resultados.

\section{REFERÊNCIAS}

1. Hirsh AT, Jensen MP, Robinson ME. Evaluation of nurses' self-insight into their pain assessment and treatment decisions. J Pain. 2010;11(5):454-61. https://doi.org/10.1016/j.jpain.2009.09.004

2. Barbosa TP, Beccaria LM, Pereira RAM. Avaliação da experiência de dor pós-operatória em pacientes de unidade de terapia intensiva. Rev Bras Ter Intensiva. 2011;23(4):470-7. https://doi.org/10.1590/S0103-507X2011000400012

3. Needham DM, Truong AD, Fan E. Technology to enhance physical rehabilitation of critically ill patients. Crit Care Med. 2009 Oct;37(10 Suppl):S436-41. https://doi.org/10.1097/CCM.0b013e3181b6fa29

4. Borges M, Oliveira LR. Fisioterapia motora em pacientes adultos em terapia intensiva. Rev Bras Ter Intensiva. 2009;21(4):446-52. https://doi.org/10.1590/S0103-507X2009000400016

5. Vincent JL, Rhodes A, Perel A, Martin GS, Della Rocca G, Vallet B, Pinsky MR, Hofer CK, Teboul JL, de Boode WP, Scolletta S, Vieillard-Baron A, De Backer D, Walley KR, Maggiorini M, Singer M. Clinical review: Update on hemodynamic monitoring-a consensus of 16. Crit Care. 2011 Aug 18;15(4):229. https://doi.org/10.1186/cc10291

6. Dias FS, Rezende EAC, Mendes CL, Silva-Jr JM, Sanches JL. Monitorização hemodinâmica em unidade de terapia intensiva: uma perspectiva do Brasil. Rev Bras Ter Intensiva. 2014;26(4):360-6. http://dx.doi.org/10.5935/0103-507X.20140055

7. Gélinas C, Fortier M, Viens C, Fillion L, Puntillo K. Pain assessment and management in critically ill intubated patients: a retrospective study. Am J Crit Care. 2004;13(2):126-35.

8. Byrd PJ, Gonzales I, Parsons V. Exploring barriers to pain management in newborn intensive care units: a pilot survey of NICU nurses. Adv Neonatal Care. 2009 Dec;9(6):299-306. https://doi.org/10.1097/ANC.0b013e3181c1ff9c 
9. Sakata KR. Analgesia e sedação em unidade de terapia intensiva. Rev Bras Anestesiol. 2010;60(6):653-8. https://doi.org/10.1590/ S0034-70942010000600012

10. Riker RR, Fraser GL. Altering intensive care sedation paradigms to improve patient outcomes. Crit Care Clin. 2009 July;25(3): 527-38, viii-ix. https://doi.org/10.1016/j.ccc.2009.05.004

11. Kumar AB, Brennan TJ. Pain assessment, sedation, and analgesic administration in the intensive care unit. Anesthesiology. 2009 Dec;111(6):1187-8. https://doi.org/10.1097/ALN.0b013e3181c0d1b5

12. Morete MC, Mofatto SC, Pereira CA, Silva AP, Odierna MT. Tradução e adaptação cultural da versão portuguesa (Brasil) da escala de dor Behavioural Pain Scale. Rev Bras Ter Intensiva. 2014;26(4):373-8. https://doi.org/10.5935/0103-507X.20140057

13. Li D, Puntillo K, Miaskowski C. A review of objective pain measures for use with critical care adult patients unable to self-report. J Pain. 2008;9(1):2-10. https://doi.org/10.1016/j.jpain.2007.08.009

14. Cade CH. Clinical tools for the assessment of pain in sedated critically ill adults. Nurs Crit Care. 2008;13(6):288-97. https://doi. org/10.1111/j.1478-5153.2008.00294.x

15. Aissaoui Y, Zeggwagh AA, Zekraoui A, Abidi K, Abouqal R. Validation of a behavioral pain scale in critically ill, sedated, and mechanically ventilated patients. Anesth Analg. 2005 Nov;101(5):1470-6. https://doi.org/10.1213/01.ANE.0000182331.68722.FF

16. Ferreira LL, Valenti VE, Vanderlei LCM. Chest physiotherapy on intracranial pressure of critically ill patients admitted to the intensive care unit: a systematic review. Rev Bras Ter Intensiva. 2013;25(4):327-33. https://doi.org/10.5935/0103-507X.20130055

17. França EE, Ferrari F, Fernandes P, Cavalcanti R, Duarte A, Martinez BP, Aquim EE, Damasceno MCP. Fisioterapia em pacientes críticos adultos: recomendações do Departamento de Fisioterapia da Associação de Medicina Intensiva Brasileira. Rev Bras Ter Intensiva. 2012;24(1):6-22. https://doi.org/10.1590/S0103-507X2012000100003

18. Cruz CT, Stumm EMF. Instrumentalização e implantação de escala para avaliação da dor em unidade de terapia intensiva neonatal: relato de casos. Rev Dor. 2015;16(3):232-4. http://dx.doi.org/10.5935/1806-0013.20150046

19. Toledo C, Garrido C, Troncoso E, Lobo SM. Effects of respiratory physiotherapy on intracranial pressure and cerebral perfusion pressure in severe traumatic brain injury patients. Rev Bras Ter Intensiva. 2008;20(4):339-43. https://doi.org/10.1590/S0103$507 X 2008000400004$

20. Hirsh AT, Jensen MP, Robinson ME. Evaluation of nurses' self-insight into their pain assessment and treatment decisions. J Pain. 2010;11(5):454-61. https://doi.org/10.1016/j.jpain.2009.09.004

21. Mendes LC, Fontenele FC, Dodt RCM, Almeida LS, Cardoso MVLML, Silva CBG. A dor no recém-nascido em unidade de terapia intensiva neonatal. Rev Enferm UFPE. 2013;7(11):6446-54. http://dx.doi.org/10.5205/reuol.3794-32322-1-ED.0711201318

22. Freitas ZMP, Pereira CU, Oliveira DMP. Escalas para avaliação de dor em neonatologia e sua relevância para a prática de enfermagem. Rev Bras Med. 2012;68(1):18-24.

23. Bassanezi BSB, Oliveira-Filho AG. Analgesia pós-operatória. Rev Col Bras Cir. 2006;33(2):116-22. https://doi.org/10.1590/S010069912006000200012

24. Silva TAMC. Controle da dor peri-operatória: revisão de literatura [Monografia]. [Curitiba]: Universidade Federal Rural do Semi-Árido; 2011

25. Falcão FL, Ribeiro FI. Avaliação da dor em recém-nascidos com distúrbios respiratórios submetidos a procedimentos fisioterapêuticos de rotina. Rev Paul Pediatr. 2007;25(1):53-8.

26. Moreira FC, Teixeira C, Savi A, Xavier R. Changes in respiratory mechanics during respiratory physiotherapy in mechanically ventilated patients. Rev Bras Ter Intensiva. 2015;27(2):155-60. https://doi.org/10.5935/0103-507X.20150027

27. Nascimento JCC, Silva LCS. Avaliação da dor em paciente sob cuidados em unidades de terapia intensiva: uma revisão de literatura. Rev Movimenta. 2014;7(2):717-20.

28. Mueller XM, Tinguely F, Tevaearai HT, Revelly JP, Chioléro R, von Segesser LK. Pain location, distribution, and intensity after cardiac surgery. Chest. 2000;118(2):391-6. https://doi.org/10.1378/chest.118.2.391 C 